\title{
From 2D to 3D super-resolution imaging through glass microspheres -INVITED
}

\author{
Stephane Perrin*, Sylvain Lecler, and Paul Montgomery \\ ICube Laboratory, CNRS - INSA de Strasbourg - University of Strasbourg, 67412 Illkirch, France
}

\begin{abstract}
Microsphere-assisted microscopy is a new imaging technique which allows the diffraction limit to be overcome using transparent microspheres. It makes it possible to reach a resolution of up to $100 \mathrm{~nm}$ in air while being label-free and full-field. An overview of the imaging technique is presented showing the influence of the photonic jet on the image nature and the unconventional behaviour of the magnification factor. Moreover, interferometry through microspheres is demonstrated for the $3 \mathrm{D}$ reconstruction of nanoelements.
\end{abstract}

\section{Introduction}

In optical imaging, observation of sub-micrometre features is a real challenge. According to theory of diffraction, the smallest discernible size through a classical microscope is of $\lambda / 2$ (where $\lambda$ is the wavelength of the broadband light source). In experiments, the resolving power appears to be reduced due to the optical aberrations, misalignments and numerical aperture of the system (NA $\leq 1.0$ in air). Since the appearance of the notion of ultramicroscopy in 1930's, several approaches have been suggested making it possible to overcome the diffraction barrier [1] such as confocal microscopy, scanning near field optical microscopy, negative-indexsuperlenses microscopy, structured illumination microscopy, and stimulated emission depletion microscopy super resolved fluorescence microscopy. However, these sub-diffraction-limit techniques are often photo-toxic for the sample, are complex to implement, need markers or require long-acquisition times.

\section{Direct imaging through microspheres}

More recently, Wang et al. experimentally demonstrated the principle of full-field super-resolution microscopy by only introducing a transparent microsphere in a classical white-light microscope [2]. This easy-to-implement and non-invasive nanoscopy technique allows the diffraction limit to be overcome by a factor of 3 ( $\lambda / 6$ in air) [3]. Through a probable conversion of the near-field information to propagating waves [4,5], microsphereassisted microscopy is thus able to achieve a lateral resolution that is superior to that attainable by confocal microscopy and by the solid immersion lens [6] and similar to that achieved from structured illumination microscopy and meta-material-based superlenses. Numerical studies and experiments have made it possible to highlight the influence of the photonic jet on the image nature (despite the performance of the photonic jet not being able to be used to predict the phenomenon behind the super resolution) $[7,8]$ and the unconventional behaviour of the magnification factor [9], respectively. Moreover, microsphere-assisted microscopy has recently been adapted for biological imaging using fluorescence [11] and dark-field imaging for label-free visualization of transparent objects [12].

\section{Interferometry through microspheres}

Furthermore, microsphere-assisted microscopy has successfully been combined to interference microscopy in

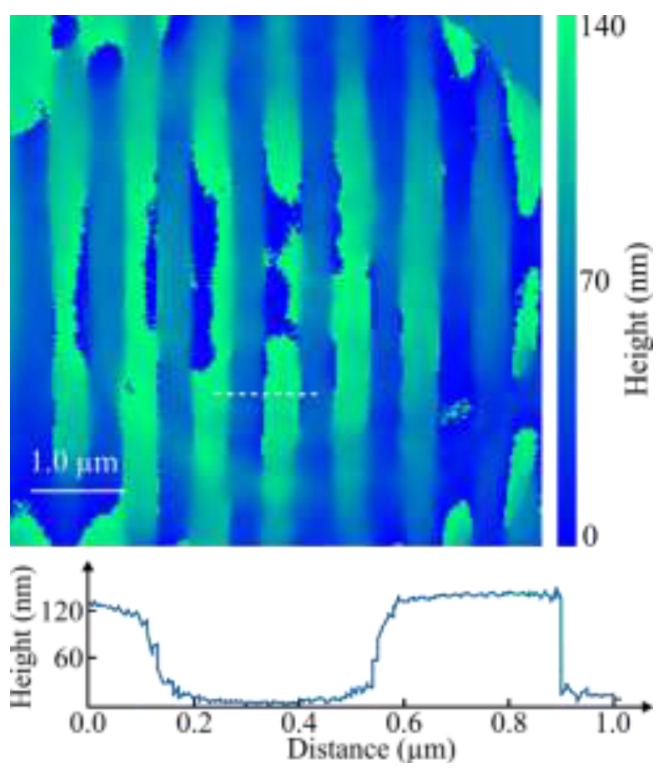

Fig. 1. Topography reconstruction of an 800-nm-period standard grating using microsphere-assisted interference microscopy compensated by two $50-\mu \mathrm{m}$-diameter microspheres. The height profile is along the dashed-white line of the high-resolution surface-topography reconstruction.

\footnotetext{
* Corresponding author: stephane.perrin@unistra.fr, ORCID: 0000-0003-2470-7636
} 
order to reconstruct surface topographies of subwavelength features $[12,13]$. Indeed, the lateral resolution in interference microscopy is improved while keeping a high axial accuracy (around $10 \mathrm{~nm}$ ). We can notice that in interference microscopy, the lateral resolution is not only limited by diffraction of light, but also by the ability of the system to retrieve the height distribution. This leads to a micrometre-scale resolution (instead of $\lambda / 2$ ). Thus, in order to further increase the 3D spatial resolution, an experimental method has recently been proposed. It consists in introducing a second microsphere in the reference arm (similar to the one in the object arm) [14]. In this way, the wave-front distortion due to the optical aberrations is avoided and the optical path difference in the interferometer arms is compensated. Topographies of 300-nm-width grating lines and 250-nm-diameter transparent nanopillars have been retrieved. In order to provide a better understanding of the phase behaviour through microspheres, a numerical model of microsphereassisted interference microscopy is being implemented.

Acknowledgements: This work received funding from SATT Conectus Alsace and was also supported by the University of Strasbourg.

\section{References}

1. Editorial, Nat. Photon. 3, 361 (2009)

2. Z. Wang, W. Guo, L. Li, B. Luk'yanchuk, A. Khan, Z. Liu, Z. Chen, and M. Hong, Nat. Commun. 2, 218 (2011)

3. V.N. Astratov, Label free super resolution microscopy (Springer, Cham, 2019)

4. Y. Ben-Aryeh, J. Opt. Soc. Am. A 33, 2284 (2016)

5. F. Wang, Y. Cao, S. Yang, X. Wang, J. Wang, T. Chen, and Y.-H. Ye, Proc. SPIE 10964, 109643H (2018)

6. A. Darafsheh, N.I. Limberopoulos, J.S. Derov, D.E. Walker, and V.N. Astratov, Appl. Phys. Lett. 104, 061117 (2014)

7. S. Lecler, S. Perrin A. Leong-Hoi, and P. Montgomery, Sci. Rep. 9, 4725 (2019)

8. A.V. Maslov and V.N. Astratov, Phys. Rev. Appl. 11, 064004 (2019)

9. S. Perrin, H. Li, S. Lecler, and P. Montgomery, Opt. Laser Technol. 114, 40 (2019)

10. H. Yang, N. Moullan, J. Auwerx, and M.A.M. Gijs, Small 10, 1712 ((2014)

11. S. Perrin, H. Li, K. Badu, T. Comparon, G. Quaranta, N. Messaddeq, N. Lemercier, P. Montgomery, J.-L. Vonesch, and S. Lecler, Phys. Status Solidi RRL 13, 1800445 (2019)

12. F. Wang, L. Liu, P. Yu, Z. Liu, H. Yu, Y. Wang, and W.J. Li, Sci. Rep. 6, 24703 (2016)

13. Lucie Hüser and Peter Lehmann, Appl. Opt. 59, 1695 (2020)
14. S. Perrin, Y.J. Donie, P. Montgomery, G. Gomard, and S. Lecler, Phys. Rev. Appl. 13, 014068 (2020) 